\title{
Advancements in Cognitive-Behavioral Modeling and Treatment of OCD
}

\author{
Neil A. Rector ${ }^{1,2}$
}

Published online: 3 April 2018

(C) International Association of Cognitive Psychotherapy 2018

I am grateful for the opportunity to bring together a number of recently completed studies that develop and test new cognitive and behavioral constructs that expand our understanding of factors relevant to the maintenance of obsessive-compulsive (O-C) cycles and their treatment. Obsessive compulsive disorder (OCD) is a debilitating disorder characterized by distressing obsessive thoughts and/or images and compulsive behaviors. OCD occurs in approximately $2 \%$ of the population, tends to be chronic with a waxing and waning of symptoms over time, and leads to a variety of significant functional impairments and decreased quality of life, establishing it as the 10th leading cause of disability worldwide. Exposure response prevention (ERP) is the most wellresearched and recognized psychotherapeutic intervention for OCD and is consistently included as a frontline treatment in clinical guidelines (NICE, 2006). Less frequently studied or well established than ERP as a treatment for OCD, the aim of cognitive therapy is to reduce obsessions and compulsions by targeting the maladaptive appraisals and dysfunctional beliefs that are implicated in the development and persistence of symptoms. The majority of meta-analyses and controlled trials have examined ERP versus cognitive therapy and found them to be equally efficacious treatments on outcome measures at post-treatment and follow-up and recent studies have increasingly tested and shown support for their integration as a combined CBT intervention. The papers included in this Special Issue address different aspects of the O-C cycle within an integrated cognitive-behavioral therapy (CBT) framework - beliefs and appraisals, safety behaviors, and developments in mindfulness-based approaches that introduce new ways of conceiving of $\mathrm{O}-\mathrm{C}$ symptom maintenance and change.

In the first article, Riskind et al. (2018) address the role of negative appraisals of unwanted intrusive thoughts, not from the customary self-perspective (e.g., being bad

\section{Neil A. Rector}

neil.rector@sunnybrook.ca

1 Department of Psychiatry, University of Toronto, Toronto, Ontario, Canada

2 Department of Psychiatry, Sunnybrook Health Sciences Centre, University of Toronto, 2075 Bayview Avenue, Toronto, Ontario M4N 3M5, Canada 
or dangerous) but from how the person experiencing the intrusion anticipates others would think of them if they knew the content of the intrusion was known-that is, the role of anticipating negative reactions/criticism from others. In a non-clinical sample, the first study examines the presence of dimensional OCD symptom scores and then examines the cross-sectional associations between standard self-oriented negative appraisals of intrusions and a newly constructed measure that examines the expected negative reactions of others. The results indicated that the anticipated social component contributes to the prediction of OCD symptom scores above and beyond self-oriented negative appraisals. A second study aimed to assess anticipated negative reactions around behaviors (which could include rituals) and found that attributing malevolent/ hostile intentions to others' behaviors also accounted for significant variance in OCD symptoms. Riskind and colleagues highlight an understudied and important social appraisal component in the $\mathrm{O}-\mathrm{C}$ cycle.

The second paper by Orr et al. (2018) aimed to test the role of excessive reassurance seeking (ERS) as a key safety-behavior in OCD and the relationship of cognitive confidence leading to the tendency to engage in repeated solicitation of safety-related information. Clinical participants with OCD were compared to nonpsychiatric controls in their self-reported tendency to seek reassurance in addition to other symptom measures and a measure of cognitive self-confidence. The results indicate that ERS is commonly reported in OCD and is associated with OCD symptom severity, particularly checking symptom severity. The results also demonstrate the ERS is associated with lower cognitive confidence, and lower decisionmaking confidence, specifically. The results of this study point to the importance of identifying and directly targeting ERS behaviors in the treatment of OCD.

The third paper by Katz et al. (2018) builds on previous cross-sectional research showing that anxiety sensitivity (AS) and obsessive beliefs relate to OCD symptom severity, and to CBT treatment outcome when examined independently, by testing whether AS and obsessive beliefs interact in the $\mathrm{O}-\mathrm{C}$ cycle to impact on clinical response. The authors highlight that based on a CBT framework, AS and obsessive beliefs influence the obsessional cycle at different stages of the path from intrusion $\rightarrow$ appraisal $\rightarrow$ distress $\rightarrow$ compulsion and therefore could be hypothesized to produce an interactive rather than additive effect within the same $\mathrm{O}-\mathrm{C}$ cycle. In patient participants receiving $\mathrm{CBT}$ for $\mathrm{OCD}$, results revealed a significant two-way interaction, whereby elevated ASI-Physical scores by elevated OBQ-Importance/Control significantly predicted higher post-treatment symptom severity following CBT. The results point to the synergy of risk variables in the O-C cycle and how treatment refinements focusing on reducing AS fears may improve treatment outcomes in CBT.

The fourth paper by Cabedo et al. (2018) provides valuable and rare data bearing on the stability of long-term treatment gains of OCD patients in routine clinical practice in community settings. Although the sample size is modest (43 completers), Cabedo et al. (2018) report encouraging evidence of the stability of long-term treatment gains at 1 and 10 years later. Results indicated that CBT was effective in decreasing OCD severity, depression, dysfunctional beliefs about obsessions, and thought-suppressing tendencies, and these changes remained stable 1 and 10 years later. These findings show that CBT for OCD can be efficaciously administered in routine clinical practice without sacrificing its efficacy or long-term positive impact. 
Finally, Selchen et al. (2018) provide an initial examination of mindfulness-based cognitive therapy (MBCT) for OCD. The authors provide an overview of the development of the MBCT for OCD treatment protocol and step-by-step procedures that introduce new opportunities to impact on the $\mathrm{O}-\mathrm{C}$ cycle. As outlined, patients can bring meta-level awareness to all aspects of the unfolding cycle; thoughts are thoughts, no matter how disturbing; emotions and sensations are transient; compulsive urges are simply urges - they need not compel action; and rituals are rituals - they neither need nor afford protection. The study directly tests the preliminary efficacy of an OCDtailored 8-week course of mindfulness-based cognitive therapy (MBCT) to treatmentseeking patients with OCD tailored to OCD either prior to receiving CBT or following a CBT intervention. The preliminary results demonstrate the potential efficacy of MBCT for OCD with large and significant reductions in obsessive-compulsive symptoms from pre- to post-treatment both as a stand-alone treatment, prior to other first-line interventions and as an augmentation treatment for patients showing only partial response to $\mathrm{CBT}$. The authors outline next steps for programmatic research for MBCT in OCD.

In this Special Issue, contributions have been drawn together that provide advancements in understanding the momentary unfolding of the obsessional cycle-new negative appraisal content focusing on social judgment/criticism/rejection; the importance of excessive reassurance-seeking as a key safety behavior; the interactive nature of negative beliefs and appraisals - anxiety sensitivity and obsessive beliefs - in predicting worse outcomes to CBT; and fresh, efficacious ways of targeting appraisals, urges, and ritualistic behavior within an MBCT framework. As presented, we hope this work will continue to inspire and promote laboratory-based and clinical research on the mechanisms relevant to the development, maintenance, and treatment of OCD.

\section{References}

Cabedo, E., Carrió, C., \& Belloch, A. (2018). Stability of treatment gains 10 years after Cognitive Behavioral Therapy for obsessive-compulsive disorder: A study in routine clinical practice. International Journal of Cognitive Therapy.

Katz, D., Laposa, J. M., \& Rector, N. A. (2018). Anxiety sensitivity, obsessive beliefs, and the prediction of CBT treatment outcome for OCD. International Journal of Cognitive Therapy.

Orr, E., McCabe, R. E., McKinnon, M. C., Rector, N. A., \& Ornstein, T. J. (2018). Excessive reassuranceseeking and cognitive confidence in obsessive-compulsive disorder. International Journal of Cognitive Therapy.

Riskind, J. H., Wright, E. C., \& Scott, M. (2018). Anticipated criticism/rejection and negative self-appraisals: Do they independently predict OCD symptoms and the negative significance of intrusive thoughts? International Journal of Cognitive Therapy.

Selchen, S., Hawley, L. L., Regev, R., Richter, P., \& Rector, N. A. (2018). Mindfulness-based cognitive therapy for obsessive compulsive disorder: Stand-alone and post-CBT augmentation approaches. International Journal of Cognitive Therapy. 